\title{
Actin Filament Turnover Regulated by Cross-linking Accounts for the Size, Shape, Location, and Number of Actin Bundles in Drosophila Bristles
}

\author{
Lewis G. Tilney, Patricia S. Connelly, Linda Ruggiero, Kelly A. Vranich, \\ and Gregory M. Guild*
}

Department of Biology, University of Pennsylvania, Philadelphia, Pennsylvania 19104-6018

Submitted March 18, 2003; Revised June 16, 2003; Accepted June 23, 2003

Monitoring Editor: Anthony Bretscher

\begin{abstract}
Drosophila bristle cells are shaped during growth by longitudinal bundles of cross-linked actin filaments attached to the plasma membrane. We used confocal and electron microscopy to examine actin bundle structure and found that during bristle elongation, snarls of uncross-linked actin filaments and small internal bundles also form in the shaft cytoplasm only to disappear within $4 \mathrm{~min}$. Thus, formation and later removal of actin filaments are prominent features of growing bristles. These transient snarls and internal bundles can be stabilized by culturing elongating bristles with jasplakinolide, a membrane-permeant inhibitor of actin filament depolymerization, resulting in enormous numbers of internal bundles and uncross-linked filaments. Examination of bundle disassembly in mutant bristles shows that plasma membrane association and cross-bridging adjacent actin filaments together inhibits depolymerization. Thus, highly cross-bridged and membrane-bound actin filaments turn over slowly and persist, whereas poorly cross-linked filaments turnover more rapidly. We argue that the selection of stable bundles relative to poorly cross-bridged filaments can account for the size, shape, number, and location of the longitudinal actin bundles in bristles. As a result, filament turnover plays an important role in regulating cytoskeleton assembly and consequently cell shape.
\end{abstract}

\section{INTRODUCTION}

We now know of a number of cell types in which the actin cytoskeleton, although complex, is invariant from cell to cell, which in turn leads to stereotypical cell shapes. Obvious examples are the microvilli on the apical surface of intestinal epithelial cells, the stereocilia on the hair cells of the cochlea, the thin filaments in sarcomeres of skeletal muscle cells, and the actin cables in Drosophila bristles. How is this achieved? Up until now the simple answer to this question was that the actin cytoskeleton is generated in sequential steps that when added together generate these complex yet nearly identical structures. A similar philosophy is used when we, as humans, build complex structures such as bridges and skyscrapers. Thus, one might predict that the components of the actin cytoskeleton in those specialized cells are made available in the right amounts at the precise moment needed for construction. Although this seems to be true in certain cases, it does not seem to be universal.

We and others have investigated the genesis of bristles as a model system to understand how complex yet reproducible actin cytoskeletons are formed during development. This system is particularly suited for such an investigation because not only is it stylized from bristle to bristle but also mutants are available that allow us to identify steps in the genesis of the cell extension. Together with the modern arsenal of ancillary cell biological techniques, the bristle has the further advantage of readily expressing changes in phe-

Article published online ahead of print. Mol. Biol. Cell 10.1091/ mbc.E03-03-0158. Article and publication date are available at www.molbiolcell.org/cgi/doi/10.1091/mbc.E03-03-0158.

* Corresponding author. E-mail address: gguild@sas.upenn.edu. notype when specific components of the actin cytoskeleton are deleted or enhanced.

In addition to sequential steps in the genesis of these complex cytoskeletal structures, what we describe herein is that rapid disassembly of certain filament clusters at each step and the assembly of others plays a regulatory role in determining the form of the final cytoskeleton. Thus, the selection of more stable assemblages over other transient assemblages ultimately prescribes the precise cytoskeleton present in these specialized cell types. In short, filament disassembly plays a role as important as assembly in the formation of cell shape. In hindsight, this is not surprising because hints of such a scenario of actin dynamics regulating cell form have been seen before in other developing systems, e.g., the cochlea (Tilney et al., 1992a) and skeletal muscle (Littlefield et al., 2001; Mardahl-Dumesnil and Fowler, 2001). In fact, in a broader context we now realize that in biological systems as opposed to human generated structures or machines, trial and error exists so that the selection, almost a Darwinian type of selection, of the most stable pattern accounts for what is found.

We report herein observations of the cytoskeleton of elongating bristles in both wild-type and mutant flies. In addition to the cortical actin cables present in fully elongated bristles, there is a population of uncross-linked actin filament snarls and small internal actin bundles in actively elongating bristles. These extracortical bundles and snarls, although prominent during development, are not found in fully mature bristles indicating that these internal bundles and snarls are eliminated during morphogenesis. In fact, using green fluorescent protein (GFP) actin, we can show that these snarls are very dynamic and display a half-life of 
$\sim 2$ min. When we treated thoraces with the membrane permeant sponge toxin jasplakinolide, a compound that stabilizes assembled actin filaments by inhibiting the "off" rate of actin subunits from a filament (Bubb et al., 1994; Bubb et al., 2000) these "extra" cortical bundles and snarls became even more prevalent and in some cases enormous in amount. Thus, by inhibiting depolymerization this toxin eliminates filament turnover so that we can, by comparing jasplakinolide-treated bristles with untreated bristles, recognize all the steps in bundle assembly, including biological mistakes and unstable actin bundles that are eliminated during the assembly of the mature actin cytoskeleton.

The results of this comparison support the notion that cross-bridging of the actin filaments into bundles, and/or attachment of the filaments to the plasma membrane stabilizes these filaments. These stabilized structures turn over at a slower rate than filaments that are either not cross-linked together at all or are cross-linked with fewer cross-bridges connecting them together. This results in large stable rib-like bundles that are attached to the plasma membrane.

Thus, the disassembly of actin filaments not only seems to be the cell's solution for correcting mistakes in bundle manufacture but also for regulating the appropriate number, size, and shape of the bundles. In short, we have begun to appreciate that the construction of a stable and well defined cell extension involves not only orchestrating elongation but also using turnover to remove unwanted bundles and to control the size and shape of wanted bundles. Thus, assembly and disassembly must be part and parcel of the same process.

\section{MATERIALS AND METHODS}

\section{Drosophila Stocks}

The Oregon-R strain of Drosophila melanogaster was used as the wild-type in these studies. The singed stock $\left(s n^{3}\right)$, the forked stock $\left(f^{36 a}\right)$, the singed-forked double mutant $\left(s n^{3} f^{36 a}\right)$, and the javelin-like $\left(j v l^{1}\right)$ stock were obtained from the Bloomington Drosophila Stock Center (Indiana University, Bloomington, IN) and maintained as viable homozygotes. A viable and fertile stock containing two copies of the wild-type forked gene and four transgenic copies (for a total of six copies per diploid female genome) was generously provided by Nancy Petersen (University of Wyoming, Laramie, WY) (Petersen et al., 1994). Stubble mutant animals (Appel et al., 1993) were constructed as transheterozygotes by using the hypomorphic allele $S b^{s b d-V X 1}$ and a deficiency Df(3R) $s b d-45$ generously provided by Ann Hammonds and Jim Fristrom (University of California, Berkeley, Berkeley, CA). A Drosophila line (B11-98) containing the GAL4driver expressed in bristles and socket cells was isolated and generously supplied by John Merriam (University of California, Los Angeles, Los Angeles, CA) and will be described in detail elsewhere (Merriam and Guild, unpublished data). The B11-98 third-chromosome was maintained over a $T M 6 B, T b$ balancer. A Drosophila line with the second chromosome containing a GFP-actin fusion under the control of a yeast UAS element (Verkhusha et al. 1999) was generously supplied by Vladislav Verkhusha (Japan Science and Technology Corporation, Kyoto, Japan) and balanced over $\mathrm{CyO}$. Animals expressing GFP-actin in their bristles were generated by crossing these two stocks and identifying GFP-expressing non-Tb white prepupae. Flies were maintained on standard cornmeal-molasses-yeast food at $25^{\circ} \mathrm{C}, 60-70 \%$ relative humidity, with a 12:12-h day/night cycle. Complete descriptions of genes and symbols can be found in Lindsley and Zimm (1992) and on FlyBase (FlyBase Consortium, 2002)

\section{Developmental Staging}

All animals were staged from the point of puparium formation, an easily recognizable and brief stage lasting $30 \mathrm{~min}$ at the beginning of metamorphosis (Bainbridge and Bownes, 1981). White prepupae were collected and placed on double-sided scotch tape in a Petri dish that was put back into the $25^{\circ} \mathrm{C}$ incubator. At the appropriate time of incubation, the Petri dish was removed and the pupae dissected.

\section{Dissection of Pupae and Culturing the Dorsal Thoracic Epithelium}

After removing the pupal case, we filleted the pupae as outlined in detail previously (Tilney et al., 1998). We carried this out under Grace's insect cell culture medium (Invitrogen, Carlsbad, CA), at $25^{\circ} \mathrm{C}$. Each fillet, which consists of the dorsal surface of the thorax, was placed on its back and very delicately the large tracheoles and fat bodies were removed with fine forceps. It is important not to clean the preparation too thoroughly (e.g., removing all the muscles and all the fat body) because it is easy to damage the underlying epidermis containing the bristles. These isolated thoracic fillets were then placed in $60 \times 15-\mathrm{mm}$ Petri plates with $5 \mathrm{ml}$ of Grace's medium with or without jasplakinolide. The jasplakinolide (Molecular Probes, Eugene, OR) was diluted into Grace's medium from concentrated stock (1 $\mathrm{mM}$ in dimethyl sulfoxide [DMSO]) to a final concentration of $3 \mu \mathrm{M}$. Control incubations using DMSO were performed in parallel. After swirling the medium in the Petri dishes to dilute the DMSO or DMSO/jasplakinolide, we added the isolated thoraces and returned the plates to the $25^{\circ} \mathrm{C}$ incubator for varying times before fixation or examination by confocal microscopy. The volume of medium was just sufficient to cover the tissue, allowing for good oxygen exchange.

\section{Confocal and Electron Microscopy}

The procedures for fixation, staining with rhodamine- or Texas Red-conjugated phalloidin, and confocal microscopy were described previously (Guild et al., 2002). The confocal images of phalloidin-stained actin bundles presented herein are typically Z-projections of several optical sections that collectively cover half the bristle diameter. Pupae expressing GFP-actin were dissected from their pupal cases at the indicated times and placed in a well on a microscope slide defined by a silicone rubber isolator (Molecular Probes), covered with a small amount of Halocarbon-700 oil (Halocarbon Products, River Edge, NJ) and a coverslip. Animals were imaged immediately using a Fluoview model BX50 confocal microscope (Olympus, Tokyo, Japan) equipped with a $60 \times$ oil immersion 1.4 numerical aperture objective. GFP fluorescence was easily detected using the 488-nm line of the argon laser set at its minimum intensity $(6 \%)$. Virtually no sample bleaching was observed under these conditions. All confocal images were processed using Adobe Photoshop (Adobe Systems, San Jose, CA) with the guidelines outlined by Murphy (2001). All images obtained from phalloidin-stained material or from GFP-labeled animals are presented as grayscale images. These images were printed as dark signals on a white background to allow for easier visualization of small actin bundles. Procedures used for thin section transmission electron microscopy have been described previously (Tilney et al., 1998).

\section{RESULTS}

During Bristle Elongation Numerous Uncross-linked Actin Filaments and Actin Bundles Form yet Disappear, Leaving Only a Limited Number of Large Stable Cortical Bundles

Examination of bristle tips in the process of elongation by using flies expressing GFP-actin or after staining with fluorescent phalloidin reveals newly formed modules that graft together to form long continuous bundles (Tilney et al., 1996). In addition, we see irregularly shaped fluorescent masses (Figure 1a). These masses were noticed in previous studies (Tilney et al., 1996; Wulfkuhle et al., 1998), but exactly where they were located and what they correspond to in thin sections were not known. Because phalloidin only binds to filaments, not monomers, these masses must be composed of a jumble of filaments. These masses often line up in rows between adjacent cortical bundles. In appropriate confocal sections, these masses are seen at the cell surface, often leading to bulges of the cell surface (arrowheads Figure 1a). As the tip continues to grow, these masses are progressively less frequently found as one moves basally from the bristle tip. In fully elongated bristles, these masses are not present (Figure 1b). In addition, we often see fluorescent threads that lie between true cortical bundles. These also disappear as bristle elongation proceeds.

To visualize these filament masses by electron microscopy, we examined thin sections through bristle tips of newly emerged and actively elongating bristles. The filament masses correspond to surface protuberances between newly formed modules that will be grafted together to form the cables. These areas contain snarls of F-actin filaments (Figure 2a, arrows). In some cases, these protuberances contain filaments that lie parallel to each other as if crossbridged together. Often these bundles lie at $90^{\circ}$ to the cor- 


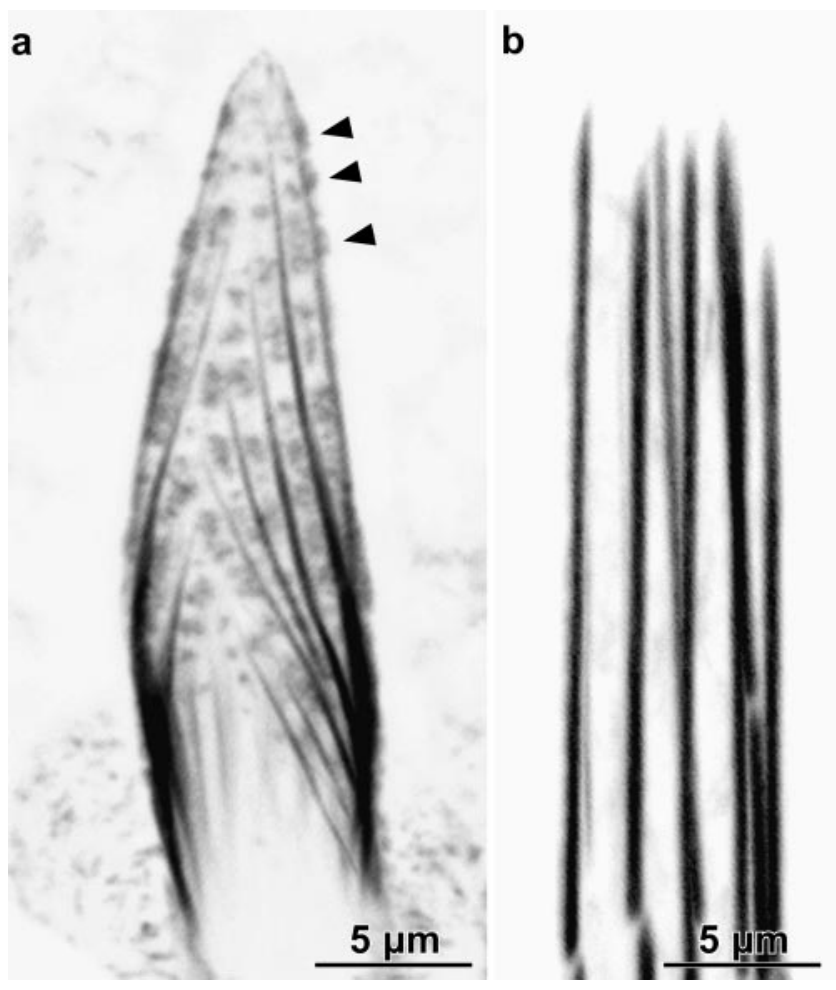

Figure 1. Confocal images through sprouting and mature bristle tips. (a) Confocal image along the surface of an elongating tip of a 33-h macrochaete stained with rhodamine-phalloidin. Arrowheads point to irregularly shaped fluorescent masses which are snarls of actin filaments. (b) Confocal image of an unusually blunt tip of a mature 48-h macrochaete from an animal expressing GFP-actin. The actin bundles are smooth and straight with no fluorescent masses in between them. Bars, $5 \mu \mathrm{m}$.

tical bundles (Figure 2b). Because these snarls disappear as elongation proceeds (Figure $1 b$ ), yet the filaments in mature cortical bundles have uniform polarity and are cross-linked together (Tilney et al., 1996), we conclude that these filament snarls disappear by depolymerization or turnover.

At the base of bristles at the same stage in bristle elongation, we also find phalloidin staining masses (Figure 3a, arrows) and numerous small internal bundles in the shaft cytoplasm (Figure 3, a and b, arrowheads). As was true at the bristle tip, the filament masses occur in our electron micrographs as surface protuberances (Figure $3 b$, arrows). The internal bundles are composed of parallel actin filaments cross-bridged together (Figure 3b, inset). Because these snarls and bundles are not present in fully elongated bristles, the noncortical bundles must be turned over just like the snarls at the bristle tip. Mutants that lack one or more cross-bridges also contain these actin snarls and surface protuberances during bristle elongation, at the elongating tips as well as all along their length (our unpublished data).

To obtain some idea how rapidly the masses illustrated in Figures 1-3 disappear as elongation proceeds, we examined the tips of sprouting bristles from pupae expressing GFPactin. We then could watch the disappearance and/or the appearance and disappearance of these masses as a function of time. One such record is illustrated in Figure 4; individual frames are separated by 2 min of elapsed time. In this figure, the arrows point to individual masses that occur in a single frame (thus occur in $<2 \mathrm{~min}$ ) and disappear completely in 2-4 min (one to two frames). From images such as this, we conclude that the masses (snarls of actin filaments) are incredibly dynamic and have a half-life of $<2 \mathrm{~min}$ ! In contrast to the dynamic behavior of the actin masses, the bundles remain intact throughout bristle elongation. One can also monitor the dynamics of the masses and/or bundles after photobleaching. These records also demonstrate that masses occur in $<2$ min (our unpublished data). These experiments will be included in a subsequent report.

\section{Further Evidence That Actin Filament Assembly and Disassembly Must Be Dynamic}

Because jasplakinolide functions to inhibit the disassembly of actin filaments (Bubb et al., 1994), any filaments that form during the treatment period are maintained as if "frozen" in time and space. Accordingly, we compared bristles cultured with and without jasplakinolide for varying time periods to get an idea of how many additional actin filaments accumulated during this period.

Within $30 \mathrm{~min}$ of incubation in jasplakinolide, we see a haze of actin in wild-type bristles that is particularly prominent in the cytoplasm at the base of the bristle (Figure 5b). This haze is not present in bristles that are not exposed to jasplakinolide, although cultured for the same time before fixation (Figure 5a). We also see small fluorescent spots in the bristles (Figure 5, b-d, arrowheads), which must correspond to the snarls of actin filaments present in our thin sections. Interestingly, the tips of the bristle fluoresce lightly and are often bulbous.

If bristles are cultured with jasplakinolide for $1 \mathrm{~h}$, we get an idea of how many more actin filaments occur with an additional $30 \mathrm{~min}$ of incubation in jasplakinolide. By $1 \mathrm{~h}$, the GFP signal within the interior of the bristle is more intense, and in some cases tiny threads (Figure $5 c$, arrow) that must correspond to internal bundles in our thin sections can be found as well as many more fluorescent spots throughout the shaft cytoplasm (Figure 5c, arrowheads). Again, the bristle tips are weakly stained and irregular in shape. By $5 \mathrm{~h}$, these internal threads and snarls are more pronounced, more numerous, and can now be found in the cell body (Figure 5d) as well as in the bristle shafts. In addition, the most basal modules elongate and extend into the cell body (Figure 4d, arrow). All of these jasplakinolide-dependent changes can also be documented by fluorescent phalloidin staining on similarly treated pupae (our unpublished data). However, because phalloidin is not membrane permeable, the thoraces cultured for timed intervals had to be fixed, permeabilized, and then stained before examination.

It is clear from these kinetic studies that internal actin filaments are assembled all the time in the absence of jasplakinolide as shown in Figures 1-3 but must be rapidly disassembled because they are only visible in the cytoplasm when frozen by this filament stabilizer.

We can get a more detailed view of the actin filaments that occur during jasplakinolide treatment by examining thin sections of wild-type bristles. What we find in addition to the cortical bundles (Figure 6a) is an enormous number of internal bundles and uncross-linked filaments (Figure 6, c and $\mathrm{d}$ ) in the shaft cytoplasm in all of our transverse sections, not just at the bristle base or tip. In some cases, the filaments in these internal bundles were hexagonally packed (Figure 6, c and d) like the cortical bundles (Figure 6b) and in longitudinal section display the $12-\mathrm{nm}$ period attributable of the fascin cross-bridge (our unpublished data). 


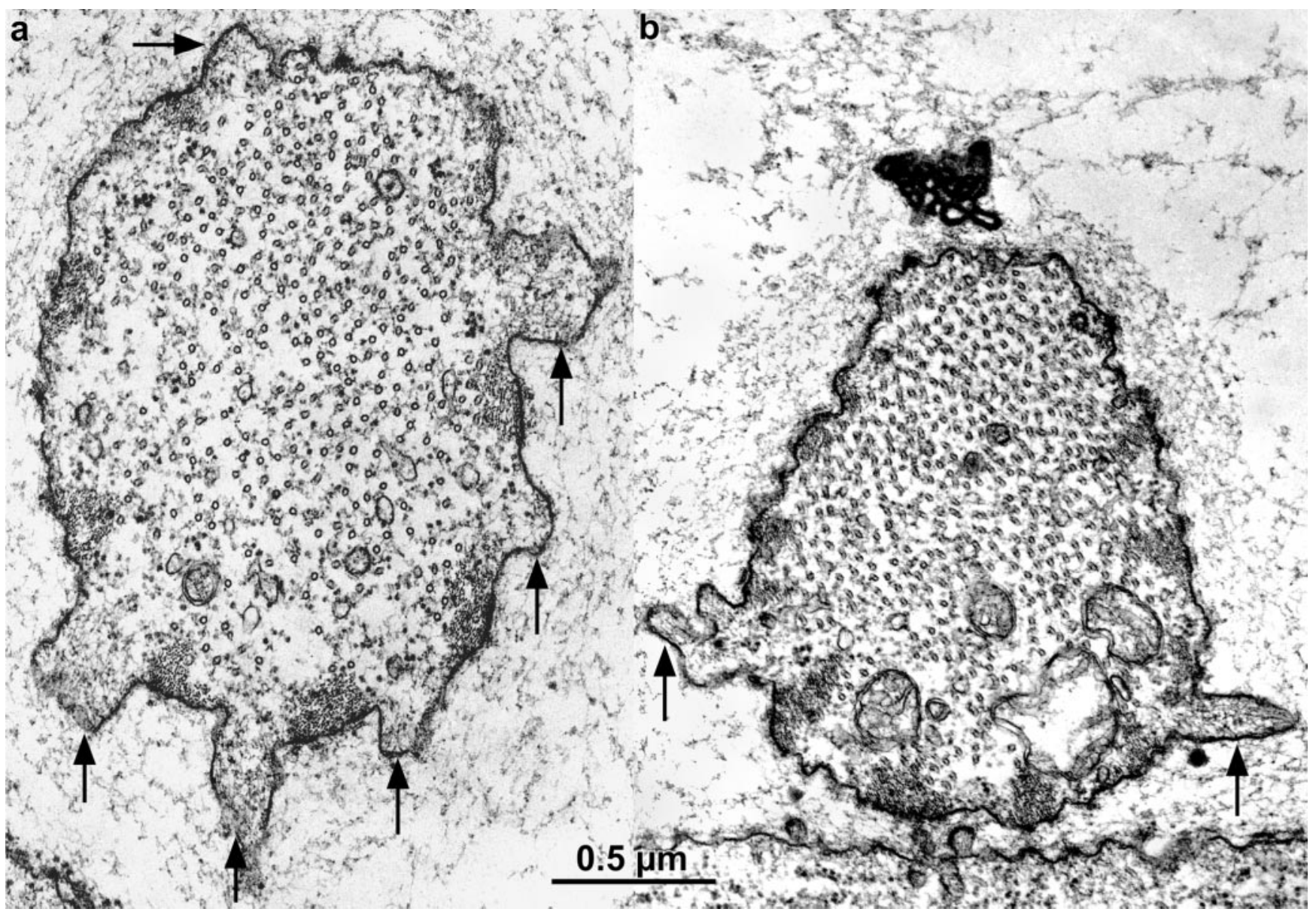

Figure 2. Thin transverse sections cut near the tip of two elongating bristles from 34-h pupae, illustrating in thin section the nature of the fluorescent masses. The arrows point to surface protuberances packed with actin filaments. In a, the actin filaments seem randomly oriented in the surface protuberances forming what we refer to as snarls, whereas in $\mathrm{b}$ the filaments tend to be clustered into nearly parallel arrays (arrows). Of interest is that these protuberances and their cortical masses of filaments are positioned between the cortical bundles that run longitudinally along the length of the bristle.

Even more spectacular are observations made on jasplakinolide-treated thoraces of mutants whose genes for the fascin and forked protein cross-bridges have been eliminated genetically. Although bristles form in this mutant, large cortical bundles of actin filaments are not present (Tilney et al., 1995). Instead, only tiny rafts of membraneattached filament clusters consisting of a filament monolayer are present (Figure 7a, some indicated by arrowheads; and c). When jasplakinolide is added to cultures of elongating bristles in this singed-forked mutant, in addition to the membrane-associated rafts (Figure 7, b and c) huge numbers of internal actin filaments occur, most of which run parallel to the longitudinal axis of the bristle (Figure 7, d-g). These internal filaments are not monolayers or rafts but exist as large clusters of actin filaments. These clusters are irregularly shaped, some containing as many as 50 actin filaments (Figure 7d). The packing of filaments in these internal bundles are liquid ordered and do not display the 12-nm period in longitudinal sections attributable to fascin. The nature of the cross-bridge used to form these liquid ordered bundles will be the topic of a future report. What is significant herein is the sheer number of filaments present during treatment with jasplakinolide.
One might obtain direct evidence for turnover of unwanted bundles as well as more accurate measurements for the time of filament turnover by monitoring internal bundle loss after washing out the jasplakinolide. We tried to do just this but like phalloidin, jasplakinolide binds to the identical site on actin (Bubb et al., 1994) and remains stabilized. In short, the jasplakinolide does not come off the bundles. One might also be concerned that jasplakinolide may not be telling us about actin filament turnover but rather produce nonphysiological "dead end" structures. Whereas a portion of the filament clusters may be dead end structures, we think that the bulk are natural intermediates because the same inappropriate snarls and internal bundles are present in bristles from thoraces cultured in the absence of jasplakinolide.

\section{Stable Bundles Do Not Contribute Actin Monomers to Bundles That Are Rapidly Turned Over}

The question immediately arose as to the source of actin monomers used to build the internal bundles and internal filaments within the bristle shaft. Do the monomers come from the breakdown of bundles that formed before incubation with jasplakinolide or from new synthesis of actin, 

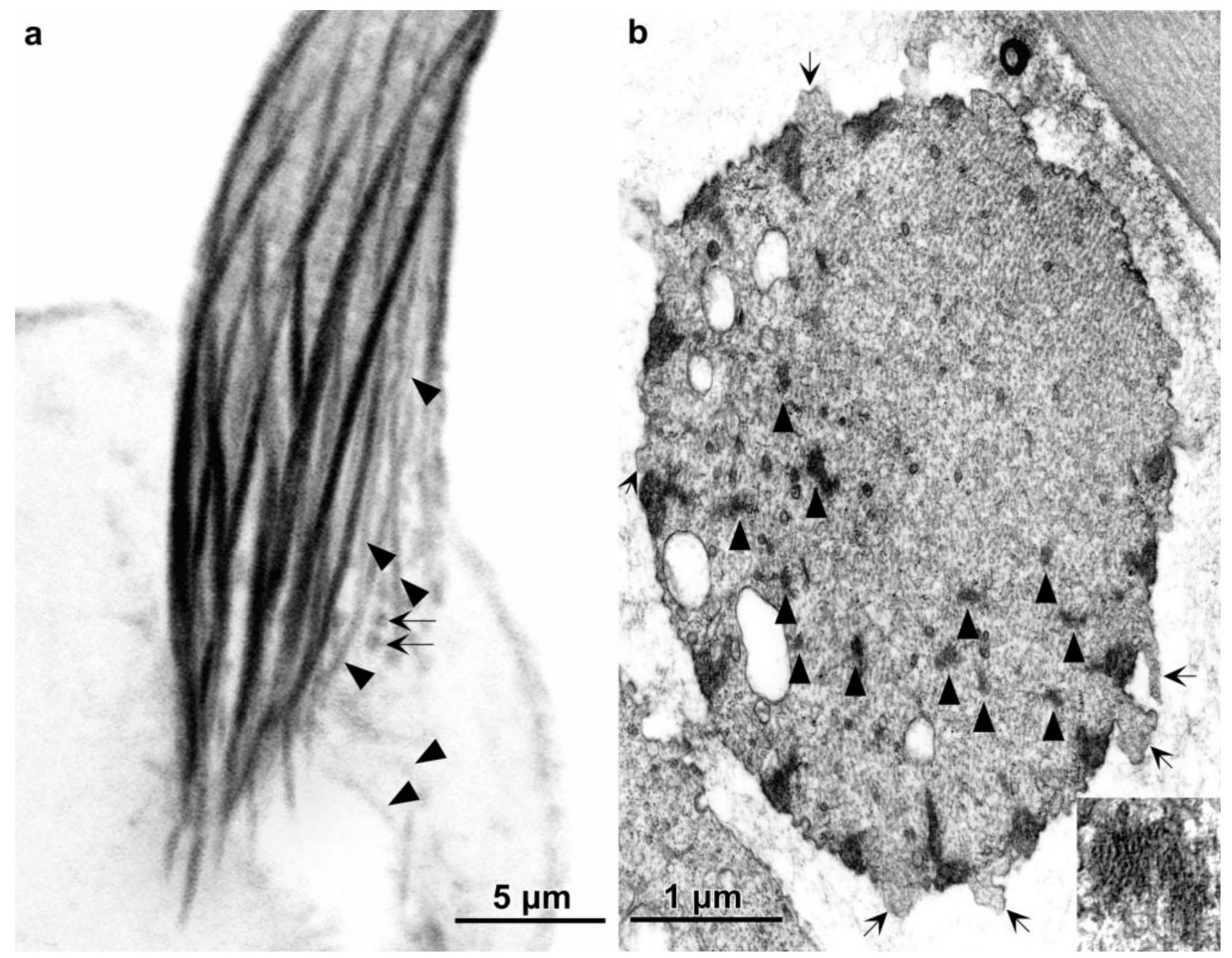

Figure 3. (a) Confocal view of a longitudinal section cut through the base of a bristle from 36-h-old pupa stained with fluorescent phalloidin. Besides the major cortical bundles are smaller bundles indicated by the arrowheads as well as small round masses (arrows). (b) Transverse section through the base of wild-type bristle from a 36-h pupa. Between some of the cortical actin bundles are surface protuberances in which are snarls of actin filaments (arrows). These are small fluorescent masses indicated by the arrows in a. The arrowheads point to some of the numerous internal bundles. One is shown at higher magnification in the inset.

which is essential for further elongation of existing bristles? To answer this question, we compared the size of the bundles present near the base of partially elongated bristles cultured in the presence of jasplakinolide. In this region, the actin bundles will have already formed before incubation in jasplakinolide. We found that these preformed bundles were comparable in size and in filament packing to those in the untreated preparations (Figure 5, a and b). Thus, the internal bundles and randomly oriented filaments present in the jasplakinolide-treated bristles must come from actin monomers that are either newly synthesized or unpolymerized before jasplakinolide treatment but not from monomers derived from the breakdown of existing cross-linked bundles.

However, we should emphasize that this jasplakinolidebased conclusion that internal bundles and randomly oriented filaments are not produced by the breakdown of existing bundles does not address whether or how frequently actin filament turnover occurs in untreated bundles. Such a conclusion is better based upon recovery after photobleach- ing experiments; these are currently in the process of being carried out.

\section{Bristles Continue to Elongate in Jasplakinolide, Even Though the Actin Bundles Are Small}

In contrast to the bristle base where internal bundles had formed before jasplakinolide treatment (Figure 3b), only tiny bundles form at the bristle tip during jasplakinolide treatment (Figure 5c). Even so, the F-actin present at the bristle tip is sufficient for elongation. In fact, bristles treated with jasplakinolide elongate $50-75 \%$ faster than untreated controls (Tilney et al., 2000b). Thus, bristle elongation does not require large actin bundles. Small bundles are sufficient, a fact that is consistent with mutants that elongate bristles with very small bundles (Tilney et al., 1995).

\section{Actin Filaments Are Stabilized against Turnover by Attachment to the Plasma Membrane}

Approximately $30 \%$ of the bristle shaft plasma membrane contacts actin bundles (Tilney et al., 2000b). This percentage 


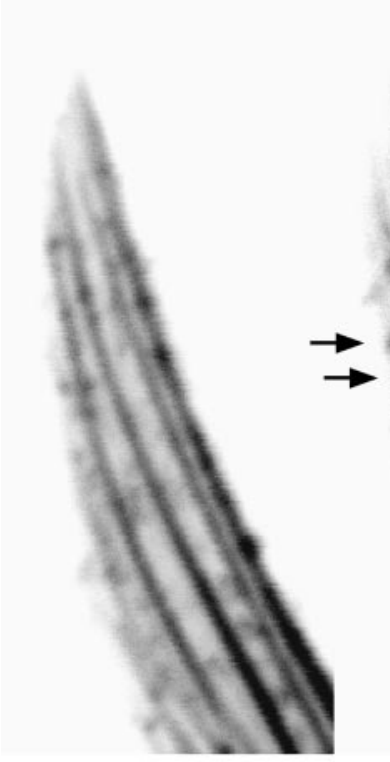

0

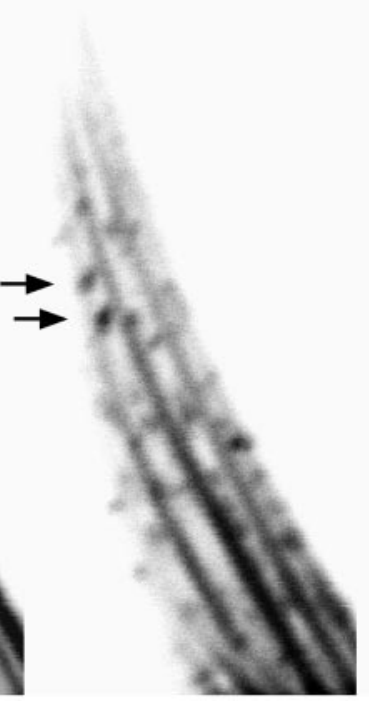

2

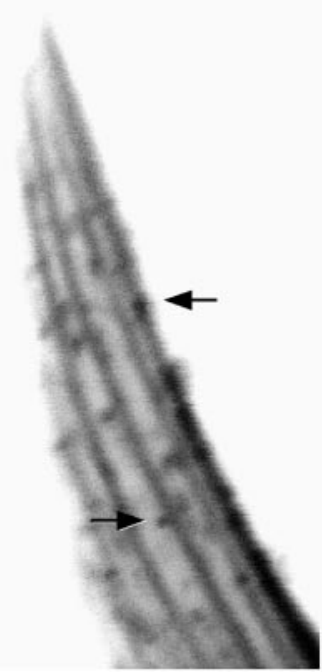

4

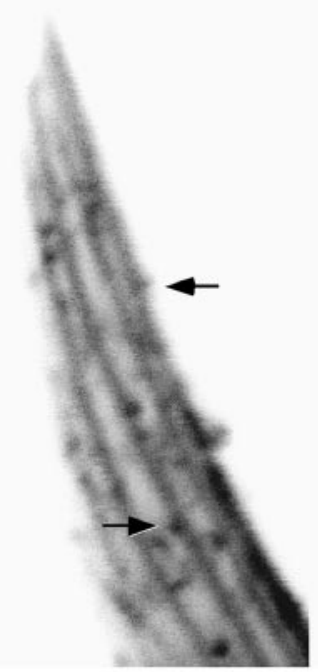

6

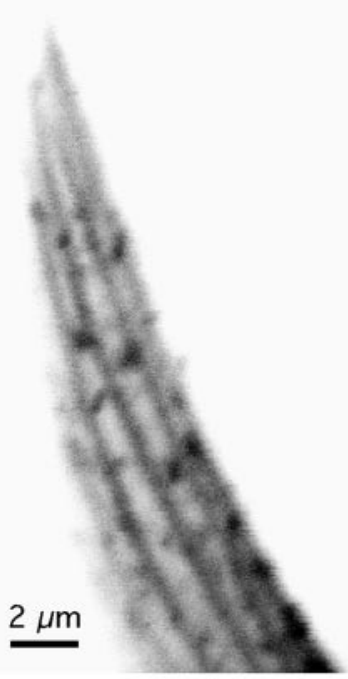

8

\section{Minutes}

Figure 4. Actin snarls in growing bristle tips are dynamic. A timed series of confocal images through a posterior scutellar macrochaete from a 31-h wild-type pupa labeled with GFP-actin. Each image represents a longitudinal section of a fraction of the bristle cylinder $(\sim 2.5 \mu \mathrm{m})$ and shows actin bundles plus many actin snarls. All snarls seem to be dynamic because individual snarls are present for only $2-4$ min, during this sequence. Several examples are indicated by arrows in the frames where they are present. Bar, $2 \mu \mathrm{m}$.

is invariant in the wild type and in a variety of mutants, which lack one or more cross-bridges or which contain additional copies of cross-bridges (Tilney et al., 2000b). Because of no diminution in the amount of surface attached to the filaments even though the bundle size is enormously reduced when one or more cross-bridges are absent in mutants, we conclude that this attachment of filaments to the membrane must stabilize these filaments.

Further evidence showing that attachment of the actin filaments to the membrane comes from examination of bristles in which the actin bundles are in the process of breakdown, which occurs once the exoskeleton is deposited. During breakdown or disassembly of the bundles, the bundles break transversely into two subbundles. The smaller portion of the former bundle remains attached to the plasma membrane (Tilney et al., 1996, Figure 4b; Guild et al., 2002, Figure 7) and separate from the larger, internal half. Significantly, the membrane attached fragment of the bundle is the last to disassemble (Guild et al., 2002, Figure 7a).

\section{Actin Filaments Are Stabilized by Cross-linking to Adjacent Filaments}

During normal microchaete development, the actin filaments are bundled into 7-11 large cortical (membrane associated) bundles with relatively few uncross-linked filaments. Because the filaments in the cortical bundles are cross-linked by fascin, the forked proteins and a third, yet to be identified cross-bridge (our unpublished data), we presume that crossbridging stabilizes the filaments in the cortical bundles relative to uncross-linked filaments. Cross-bridging then could account for the stability of filaments, a fact consistent with in vitro studies (Zigmond et al., 1992). Why don't we see tiny cortical bundles in bristles of untreated thoraces?
The answer to this question comes from carefully examining the cortical actin bundles. Let us begin by considering a maximally cross-linked bundle that we encounter in a wild-type bristle. The filaments on one side of the bundle are attached to the plasma membrane and thereby stabilized (Figure 8a). Accordingly, they are represented as filled circles in Figure 9. In the bundle proper, each maximally crosslinked filament will be connected to all six of its neighbors and thus stabilized. On the other hand, filaments at the periphery of the bundle have fewer connections, and in some cases only 1 . Thus, the core filaments in the center of the bundle should be stable (Figure 9, filled circles), whereas the peripheral filaments will be less stable because they are cross-linked less extensively (Figure 9, open circles). For a small bundle (Figure 9a), this means that 11 of 28 filaments are labile, or $40 \%$, but for larger bundles (Figure 9b) only 16 of 53 filaments are labile, or $30 \%$. Accordingly, larger bundles with relatively smaller edges would tend to be more stable than smaller bundles because filament turnover would be minimized. As the number of filaments in a bundle increases, the fraction of those filaments located in the core increases (Figure 9c).

\section{Cross-Linker Abundance Predicts Bundle Size and Shape}

Now let us examine actin bundles in a variety of mutants and see whether our concept of rapid turnover of inadequately cross-linked actin filaments relates to final bundle size and shape. This can most dramatically be done by comparing actin bundles in wild-type and mutant bristles all reproduced at the same magnification (Figure 8 ). We selected individual bundles from approximately the same distance from the bristle base (determined by the bristle diameter in thin section as the bristle tapers from its base to its 


\section{cultured $1 \mathrm{hr}$}

a

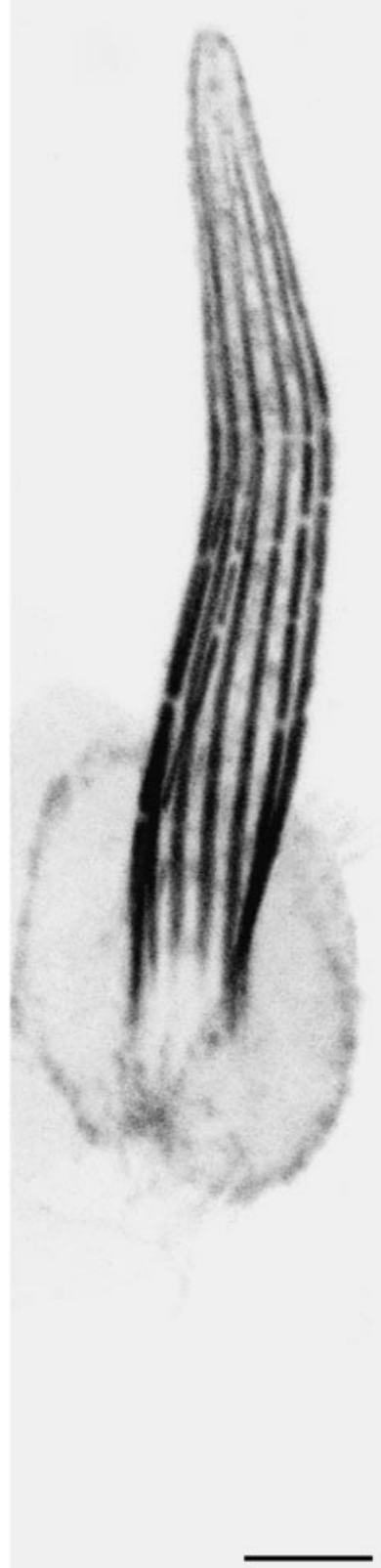

Jasp. $30 \mathrm{~min}$

b

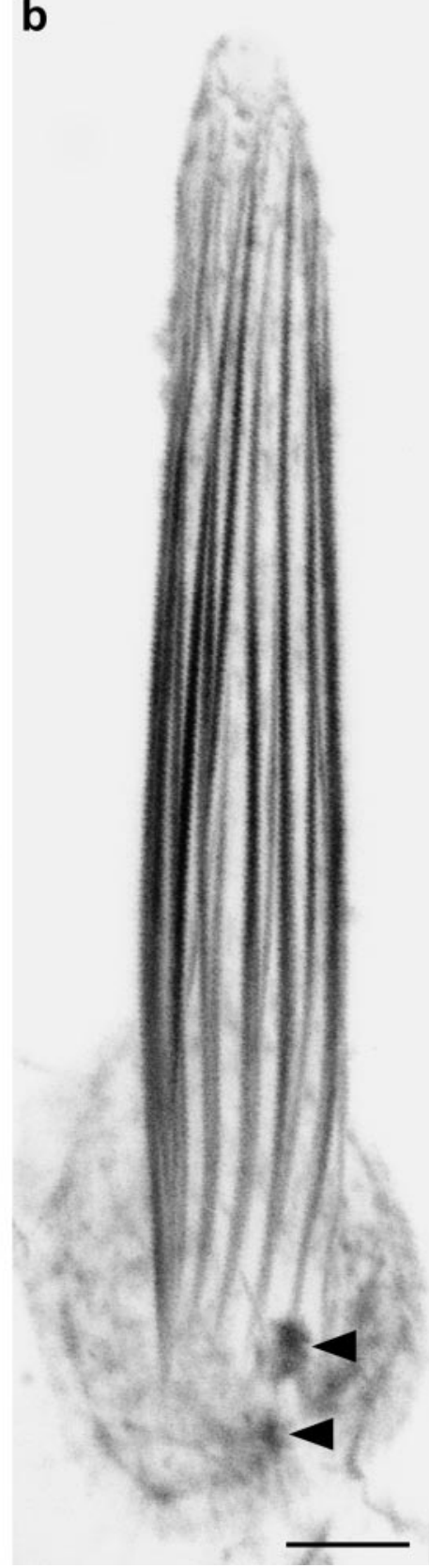

Jasp. $1 \mathrm{hr}$

C

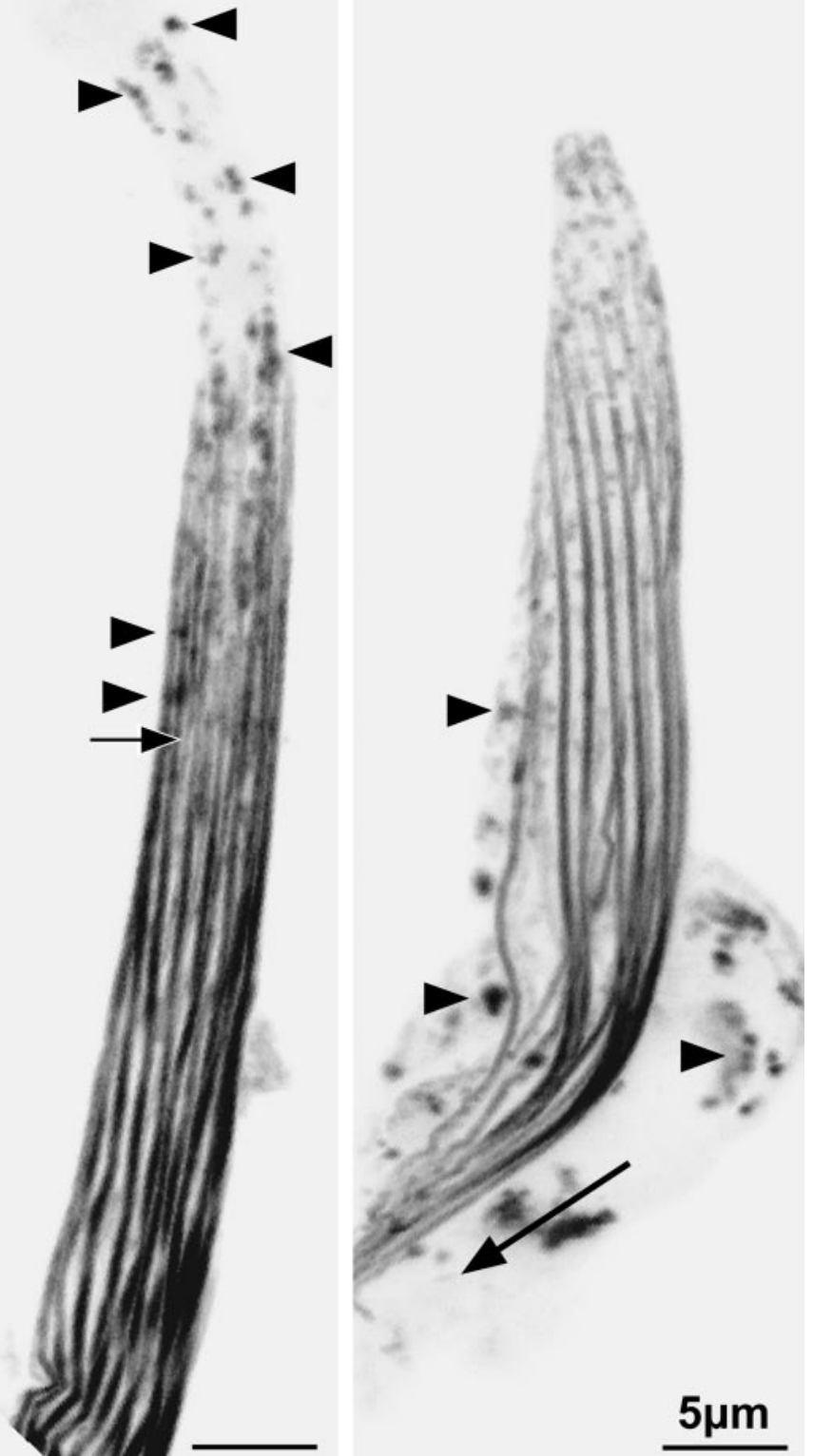

Figure 5. Confocal views through bristles from animals expressing GFP-actin and cultured in the presence of jasplakinolide for increasing times. (a) Confocal image through a 37-h macrochaete cultured for $1 \mathrm{~h}$ after dissection (control). (b) Confocal image through a 37-h pupal macrochaete cultured for $30 \mathrm{~min}$ in the presence of $3 \mu \mathrm{M}$ jasplakinolide. At the bristle base (arrowheads) fluorescent snarls are seen which are not present in a. In addition, at the base are tiny fluorescent threads. (c) Confocal image through the shaft region of a 37-h pupal macrochaete cultured for $1 \mathrm{~h}$ in the presence of jasplakinolide. Many actin snarls can be seen throughout the shaft as well as toward the tip (arrowheads). Notice that the tip does not contain continuous bundles of actin filaments. The arrow points to a thin internal bundle. (d) Confocal image through a region of a 37-h pupal macrochaete cultured for $5 \mathrm{~h}$ in the presence of jasplakinolide. Numerous actin snarls stabilized by jasplakinolide can be seen throughout the shaft and in the cell body (arrowheads). In addition, the basal ends of the actin bundles have elongated into the cell body (arrow).

tip) and at the same position on the bristle circumference (because the bundle size is smaller on the superior edge relative to the inferior edge; our unpublished data).
Such a comparison of bristle bundles reveals a number of interesting aspects. First, all seven bundles depicted in Figure 8 seem to have similarly sized surfaces attached to the 


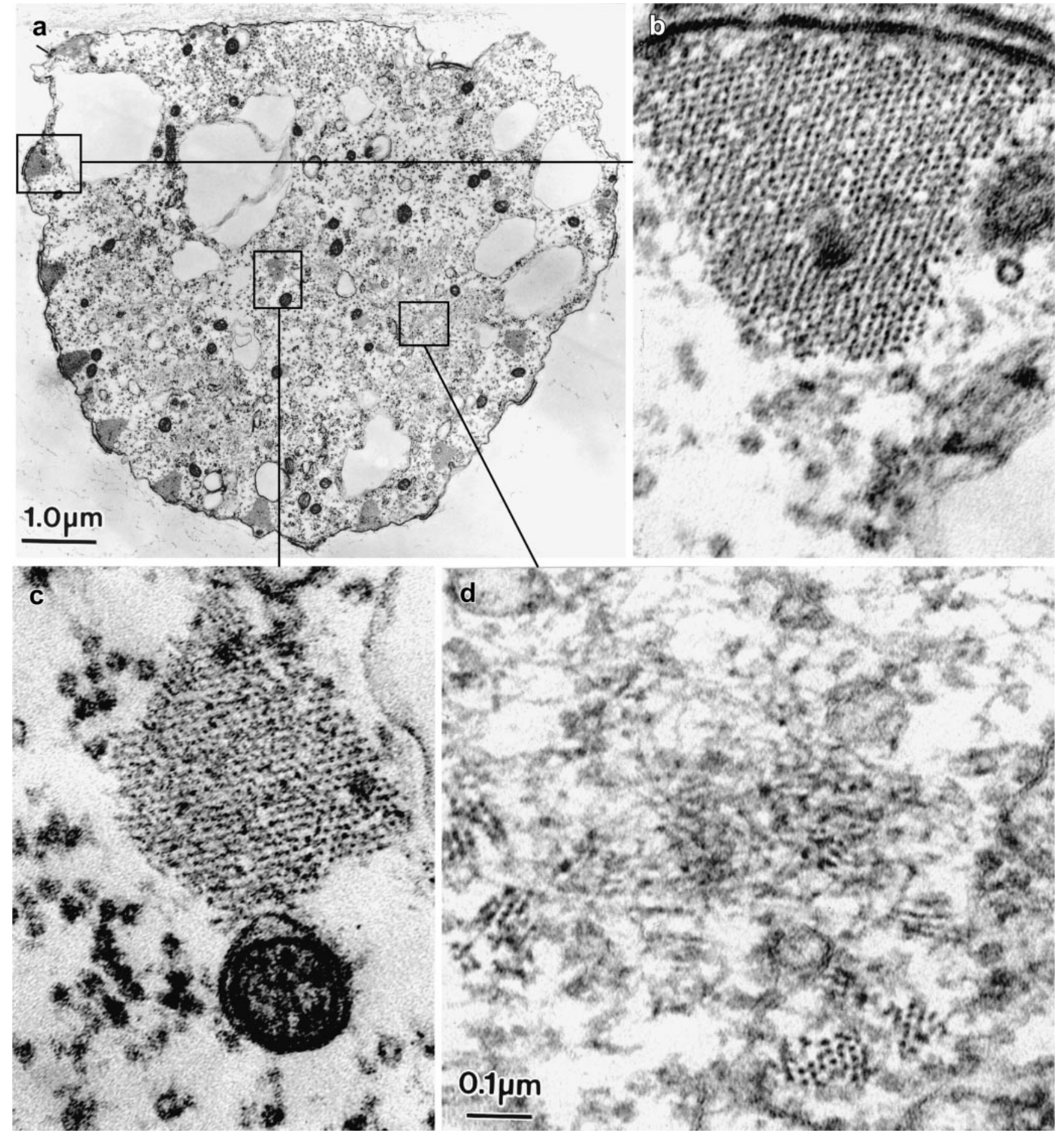

Figure 6. (a) Transverse section through a microchaete from a 41-h pupa cultured in jasplakinolide for $5 \mathrm{~h}$. This section is cut near the bristle base so the large cortical bundles formed before incubation in jasplakinolide. (b) Higher magnification of a cortical bundle boxed in a. Of interest here is that this bundle is composed of a hexagonally packed array of actin filaments indistinguishable from cortical bundles in bristles that had not been exposed to jasplakinolide. (c) Higher magnification of an internal bundle seen in the shaft cytoplasm of the jasplakinolide-treated bristle section depicted in a. Note that this bundle is composed of hexagonally packed actin filaments. (d) Also present in the shaft cytoplasm are randomly arrayed uncross-linked filaments and tiny cross-linked bundles. Bars, $1 \mu \mathrm{m}(\mathrm{a}) ; 0.1 \mu \mathrm{m}$ (b-d).

plasma membrane, as we would predict from previous studies (Tilney et al., 2000b), and what we stated earlier even though the bundles vary dramatically in cross-sectional area or number of filaments. This observation makes sense be- cause the filaments attached to the plasma membrane seem to be stable and the percentage of cell surface with attached bundles is constant. Second, if one or more cross-bridge is absent the bundle size is dramatically reduced as seen in the 

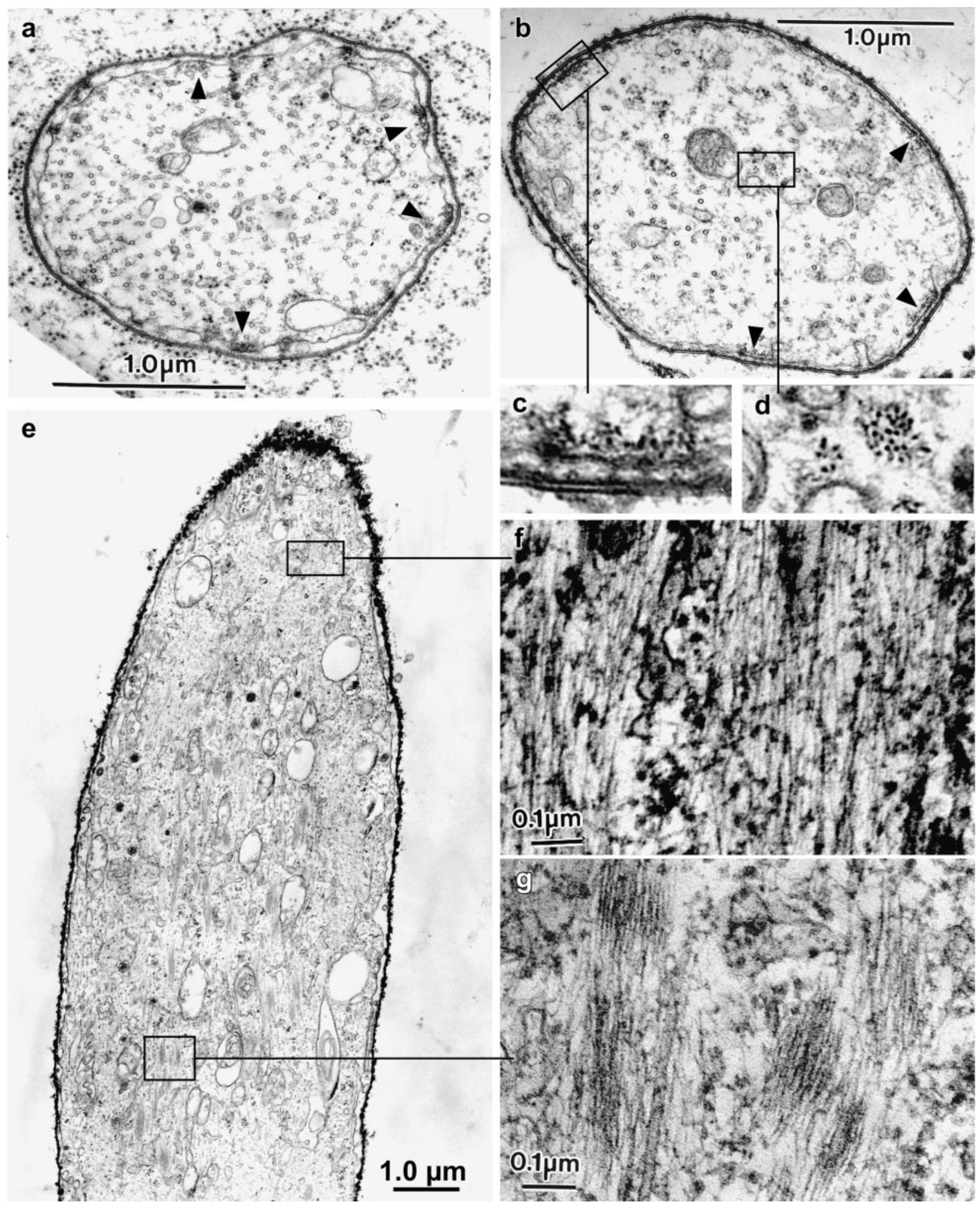

Figure 7. (a) Transverse section through a bristle of a 41-h singed-forked mutant pupa. This mutant lacks the actin cross-linkers fascin and forked. Attached to the plasma membrane that lies just inside the developing exoskeleton (showing as two dense lines in this micrograph) are tiny rafts of actin filaments. Some of these rafts are indicated by the small arrowheads. No other actin filaments are visible in this micrograph, although the center of the bristle shaft has a population of microtubules. (b-e) Sections through 36-h singed-forked mutant pupal bristles cultured for $5 \mathrm{~h}$ in jasplakinolide. (b) Transverse section. Attached to the plasma membrane are tiny rafts of actin filaments similar to what is indicated in a. Some of these are indicated by small arrowheads. One is boxed and shown at a higher magnification in c. In addition, small internal bundles are seen in the cytoplasm. One of these is boxed and shown at higher magnification in $\mathrm{d}$. (e) Longitudinal section. There is a large population of internal filaments both in bundles and as free filaments. Some of these areas indicated by the boxes are shown in higher magnification in $\mathrm{f}$ and $\mathrm{g}$. Bars, $1 \mu \mathrm{m}(\mathrm{a}, \mathrm{b}$, and $\mathrm{e}) ; 0.1 \mu \mathrm{m}$ ( $\mathrm{f}$ and $\mathrm{g})$. 


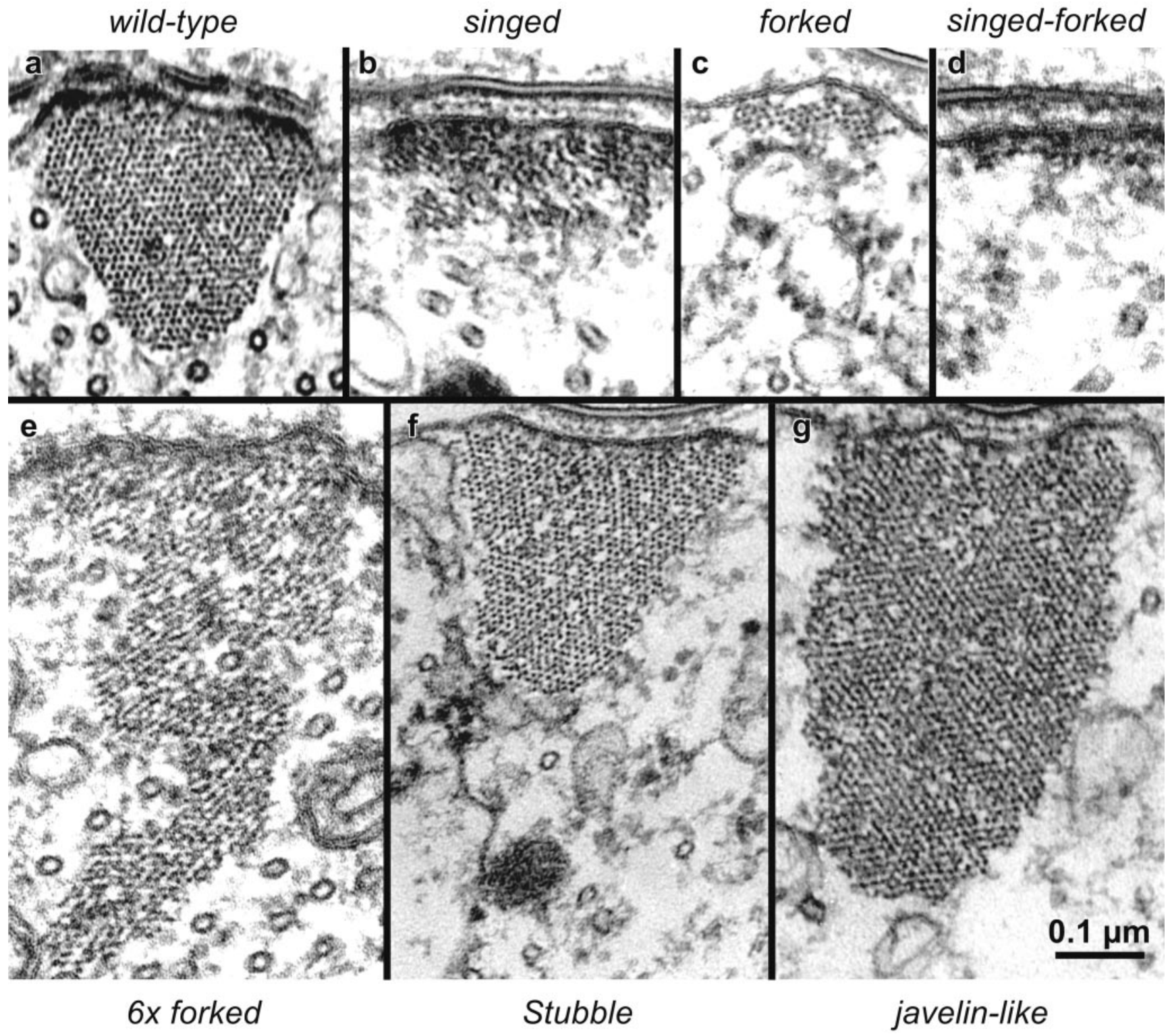

Figure 8. The concentration of actin filament cross-bridges can determine bundle cross-sectional shape. Representative thin section images of cortical membrane-associated actin bundles in wild-type and mutant microchaetes. All images shown at the same magnification. (a) Wild-type bundles exhibit normal cross-sectional profile in transverse section. Note the triangular shape with a slightly rounded cytoplasmic vertex. (b) The singed mutant bundle forms without the fascin cross-bridge and exhibits a rectangular shape flattened against the plasma membrane. (c) The forked mutant bundle forms without the forked cross-linker and also exhibits a flattened rectangular shape with fewer filaments than singed bundles. (d) The singed-forked double-mutant exhibits an extreme flat, pancake profile composed of a monolayer of filaments attached to the plasma membrane. (e) A bundle formed in the presence of additional forked cross-bridges $(6 \times$ forked $)$ contains more filaments than the wild-type and exhibits an extremely "tall" isosceles-like triangle profile that penetrates deep into the bristle shaft cytoplasm. (f) The Stubble mutant exhibits a triangular cross-sectional profile that is larger than wild type. (g) The javelin-like mutant exhibits huge bundles that show rectangular profile in cross-section that extend deeply into the cytoplasm. Of significance is the fact that even though the bundles have very different shapes, the side of the bundle attached to the plasma membrane is approximately the same length in all cases. Bars, $0.1 \mu \mathrm{m}$.

mutant lacking fascin (Figure $8 \mathrm{~b}$ ) (the singed mutant) or lacking the forked proteins (Figure 8c) (the forked mutant) or lacking both fascin and the forked proteins (the forked proteins are homologs of espin; Bartles et al., 1998) (Figure 8d) (the singed-forked double mutant). This observation also makes sense because bundles with fewer cross-linkers will be smaller because the filaments making up the bundles are less stable. Third, if cross-linker concentration is increased the bundles become larger and thus more stable. This is the case in flies that overproduce the forked proteins (Figure 8e) and in a Stubble mutant, a mutant lacking a certain class of transmembrane protein (Appel et al., 1993) (Figure 8f), where bristle length is reduced by $35 \%$ yet the same amount of components is present (our unpublished data). Fourth, filament packing in the bundles depends upon what crossbridges are added or removed. If fascin is eliminated (Figure 


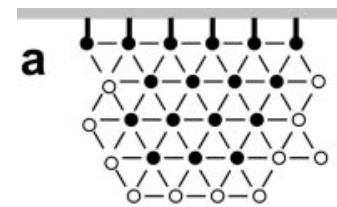

$11 / 28$

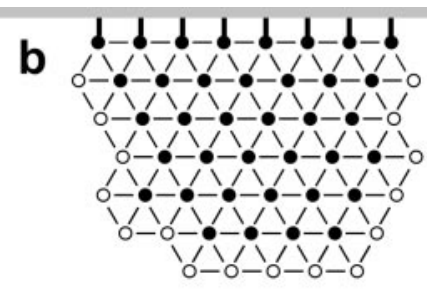

$16 / 53$

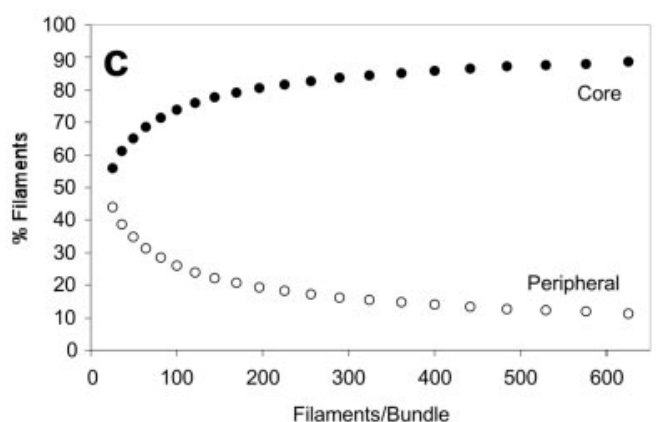

Figure 9. Increasing bundle size increases the proportion of maximally cross-linked actin filaments. (a) Drawing depicting a hypothetical bundle of 28 actin filaments. Those filaments which are either maximally cross-bridged together (six cross-links) or stabilized by attachment (bold cross-link) to the plasma membrane (horizontal gray line) are shown as closed circles. Those filaments at the periphery of the bundle are less cross-bridged and more liable to turnover are shown as open circles. In this small bundle $39 \%$ of the filaments (11/28) are liable for turnover. (b) Drawing of a hypothetical bundle of 53 actin filaments. In this larger bundle, only 30\% of the filaments (16/53) are liable for turnover. (c) Graph illustrating that bundles of increasing size have proportionally fewer imperfectly cross-linked filaments. We modeled square-like bundles (such as those in a and b) with increasing numbers of filaments on each edge (5-25) to generate hypothetical bundles containing 25-625 filaments. In each case, we calculated the percentage of peripheral filaments on the cytoplasmic edge of the bundles (open circles) and the percentage of core filaments maximally cross-linked to one another or attached to the plasma membrane (closed circles) relative to bundle size.

$8 \mathrm{~b})$, the filaments become liquid ordered and not hexagonally packed. Alternatively, if the forked proteins are eliminated (Figure 8c) fascin remains and the filaments are hexagonally packed like wild-type bundles (Figure 8a). If additional forked protein is present along with wild-type amounts of fascin, portions of the bundle exhibit hexagonally packed filaments, whereas other portions display liquid-ordered filaments (Figure 8e). And finally, in the Stubble mutant and in the javelin-like mutant (the protein affected here is still unknown), the filament packing resembles that of the wild type by being hexagonally packed (Figure $8, \mathrm{f}$ and $\mathrm{g}$ ).

In short, our data on mutant bristles lend support to our conclusion that cross-bridging stabilizes actin filaments against turnover. When cross-bridging is maximized, bundle size will be large, if actin-actin cross-bridging is reduced, i.e., in the singed or forked mutants, bundle size will be reduced. In the extreme case where both fascin and the forked proteins are eliminated, only rafts of filaments are present, stabilized solely because they are attached to the plasma membrane. Alternatively, small nonmembrane associated bundles will also turnover because not only is there no membrane to stabilize them but also there would tend to be fewer cross-bridges between filaments.

\section{Actin Filament Pointed Ends Are Capped as Modules Mature}

We concluded that filaments cross-bridged together and/or attached to the membrane are more stable than uncrosslinked filaments or internal filaments. In principle, additional stability can be conferred on filaments if they are capped on one or both ends. We have demonstrated that the barbed ends of the filaments in the modules are dynamic and thus modules can elongate and shrink (Guild et al., 2002). What about the pointed end of the filaments in the modules; are they uncapped as well?

To test this possibility, we compared the ends of the actin bundles at the bristle base in the socket region in the presence or absence of jasplakinolide. Because the basal ends of each module are the pointed ends of the filaments (Tilney et al., 1996), if these ends elongate and are then stabilized by jasplakinolide then the pointed ends cannot be capped. When we examine thoraces isolated from young pupae (33-37 h old) cultured in the presence of jasplakinolide for 30 min (Figure 10b), $5 \mathrm{~h}$ (Figure 10c), or $8 \mathrm{~h}$ (our unpublished data), we indeed found that the basal ends of the actin bundles elongated beyond the base of the bristle shaft and deep into the cell body. This was not the case for incubations in the absence of jasplakinolide (Figure 10a). However, if we isolated the thoraces from older pupae, e.g., $41 \mathrm{~h}$, and incubated them with jasplakinolide for $1 \mathrm{~h}$ (Figure 10d) or $5 \mathrm{~h}$ (our unpublished data), the basal ends of the bundles failed to elongate and looked exactly like typical basal bundles that terminate with flat ends. Accordingly, by $41 \mathrm{~h}$ the pointed ends of the filaments seem capped. This conclusion was arrived at independently by our in vitro studies on filament disassembly after the bristles have reached their final length (Guild et al., 2002). Thus, capping of the pointed ends of older bundles would additionally stabilize them, but newly formed bundles that may be imperfectly cross-bridged and/or lack membrane attachment will be turned over.

We interpret our observation (Figure 10) that jasplakinolide induces pointed end growth early in bristle elongation, but not late, as evidence for late pointed end capping. Other interpretations, including actin bundle retrograde flow, diminution of G-actin supply, and conversion from transient to permanent capping are possible but seem less likely for the following reasons. First, from Figure 10b, we can calculate that pointed end growth can occur at $\sim 32 \mu \mathrm{m} / \mathrm{h}$. This is almost 10 times faster than the retrograde flow rate measured by Fei et al. (2002). Second, the idea that actin monomer concentration becomes limiting after $41 \mathrm{~h}$ also seems unlikely because bristle tips are elongating with exponential growth at this time (Tilney et al., 2000a). In addition, these cells contained an additional copy of an actin-GFP gene fusion that should result in an increase in G-actin concentration in these cells. And finally, converting a transient pointed end capper to a permanent cap, as per Mardahl-Dumesnil and Fowler (2001), has the same functional outcome, namely, the pointed ends become capped after $41 \mathrm{~h}$. 

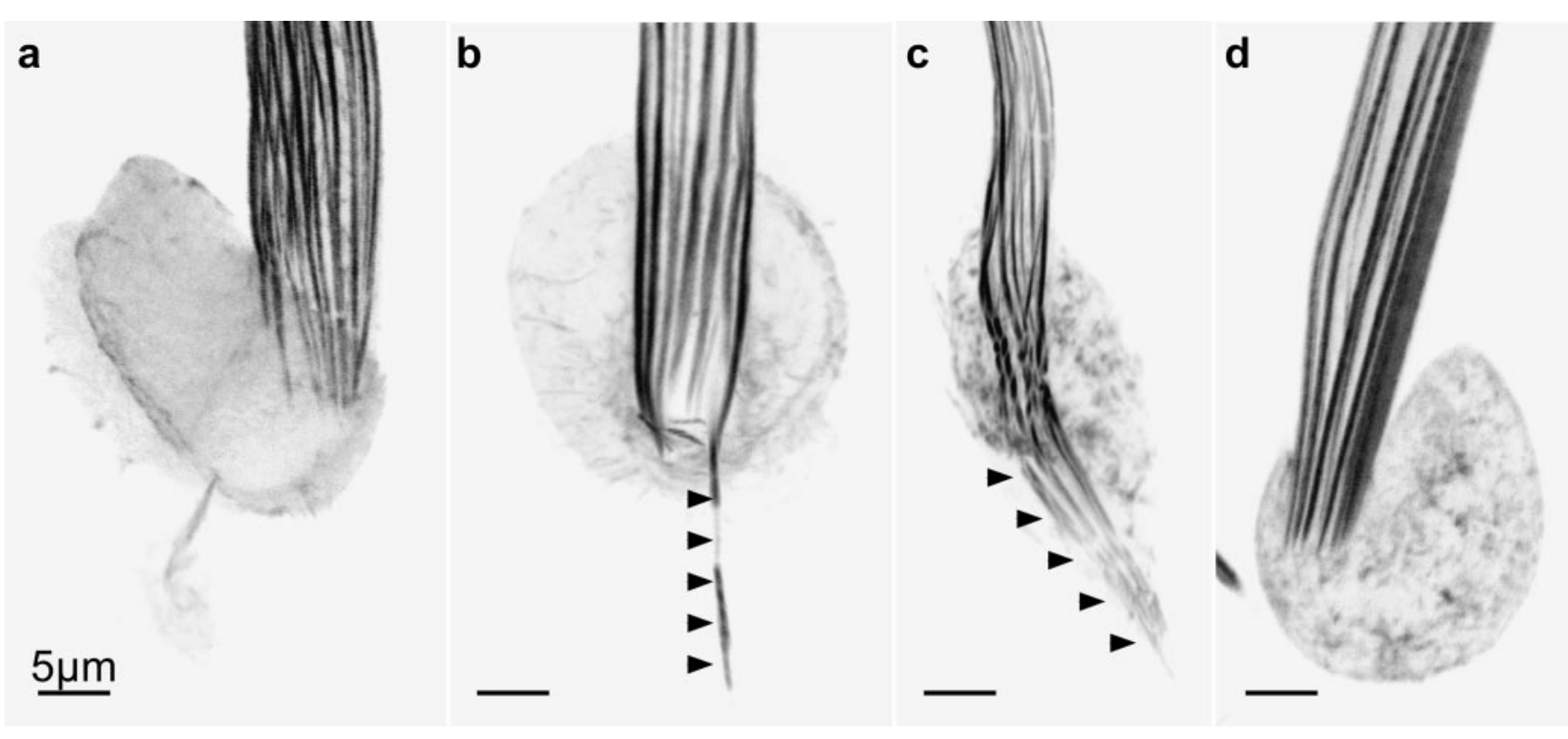

Figure 10. Evidence for capping of actin filament pointed ends at the base of modules in older bristles. Confocal views through bristles from animals expressing GFP-actin. (a) Confocal image through the socket region of a 37-h pupal macrochaete cultured for $1 \mathrm{~h}$ in the absence of any drug (control). Actin bundles terminate normally at the base of the shaft. (b) Confocal image through the socket region of a 37-h pupal macrochaete cultured for $30 \mathrm{~min}$ in the presence of $3 \mu \mathrm{M}$ jasplakinolide. At least one actin bundle has elongated into the cell body and been stabilized by jasplakinolide (arrowheads). (c) Confocal image through the socket region of a small 37-h pupal macrochaete cultured for $5 \mathrm{~h}$ in the presence of jasplakinolide. Many actin bundles have elongated into the cell body and have been stabilized by jasplakinolide (arrowheads). The discontinuous appearance of some of the bundles in the shaft above the arrowheads is an artifact of inadequate Z-sampling through an unusually thick and vertical region of this cell. (d) Confocal image through a region of a 41-h pupa cultured for $1 \mathrm{~h}$ in the presence of jasplakinolide. Actin bundles terminate normally at the base of the shaft. Bristle tips are up. Bars, $5 \mu \mathrm{m}$.

\section{DISCUSSION}

\section{Actin Filaments in Bundles Being Maximally Cross-linked and/or Attached to the Plasma Membrane Are Stable Yet Uncross-linked or Poorly Cross-linked Filaments Are Unstable and Subsequently Depolymerize}

Irregular masses of polymerized actin filaments can be seen at the tip of newly emerging bristles (Tilney et al., 1996; Wulfkuhle et al., 1998). In thin section, these masses are snarls of randomly organized actin filaments located in membrane protuberances that extend from the surface of the bristle shaft between the cortical actin bundles. As the bristle elongates to form bona fide modules and then cables of bundled actin filaments, these masses of uncross-linked filaments are reduced in size and number and ultimately disappear. Similar events are found at the bristle base. In newly emerging microchaetes, in addition to the 10 to 11 cortical bundles, we find a large number of small internal bundles as well as the snarled masses in protuberances. As elongation proceeds, these internal bundles and cortical masses gradually disappear. Thus, during normal bristle elongation there is a transient population of internal bundles and filament masses that form and then disappear, leaving only the large cortical bundles.

When thoraces with elongating bristles are cultured in the presence of jasplakinolide, both the number of internal bundles and uncross-linked filaments increase dramatically relative to untreated cultured thoraces. Furthermore, the number of internal bundles and uncross-linked filaments increases with the time of jasplakinolide treatment. Because jasplakinolide stabilizes actin filaments by inhibiting actin filament disassembly (Bubb et al., 1994, 2000), these internal bundles of uncross-linked actin filaments that have assembled during jasplakinolide treatment are in essence frozen in place. We also demonstrated that the actin monomers for the massive increase in internal bundles and cross-linked filaments are not derived from the membrane-associated actin bundles that existed before jasplakinolide treatment but must be polymerized from previously unpolymerized monomers. This was particularly dramatic in the singedforked double mutant where only tiny rafts of filaments are seen in the untreated mutant bristles but after jasplakinolide treatment the shaft of the cytoplasm fills up with assembled filaments. From all these observations, we conclude that internal bundles and uncross-linked filaments, both of which are seen in untreated bristles, are labile and disassemble shortly after assembly. Jasplakinolide, by freezing all filaments so that they cannot disassemble, shows us how dynamic the noncortical bundles must be.

In contrast, membrane-associated cortical cables seem stable and do not turn over. The best evidence for this apparent stability comes from the in vivo observations of the bundles using GFP-labeled actin (Guild et al., 2002). Further evidence documented in this report is that the maximally crossbridged and membrane associated filaments in the actin cables are capped on their pointed ends, at least in pupae $41 \mathrm{~h}$ of age. Finally, Guild et al. (2002) showed that cytochalasin leads to the breaking up of the cortical cable bundles into modules and submodules but that overall disassembly of the filaments in the modules was exceedingly slow, taking many hours. We presented evidence in this article that this stability of the large cortical bundles is achieved by maximally cross-bridging the filaments to each other and to the 
plasma membrane. Thus, internal bundles and/or free filaments are eliminated during bristle morphogenesis by turnover as a result of being poorly cross-bridged or of a lack of membrane association.

Turnover also helps us to understand why bristles treated with jasplakinolide elongate faster, usually $50 \%$ faster than bristles on untreated thoraces. Because all filaments that polymerize are frozen in place after jasplakinolide treatment, the overall rate of growth will be faster if turnover is eliminated because small bundles, or even free filaments, can act to drive cell elongation. Even so, the resultant bristles are somewhat different from the wild-type bristle. By analogy, our mutant pupae that lack one or more cross-bridges, e.g., the forked or singed mutants, elongate bristles that seem twisted, e.g., singed mutants, or bent, e.g., forked or wimpy, e.g., the singed-forked double mutant. To form large maximally cross-bridged bundles takes more time which puts a "brake" on elongation, albeit the final cell extension is of defined length and rigidity.

\section{How Actin Dynamics Could Influence the Number of Actin Cables}

In a previous article (Tilney et al., 2000b), we demonstrated in the wild type and in mutants that $30 \%$ of the plasma membrane of each bristle is allocated to bind to the filaments of the cortical cables. Because each bristle tapers, this means that in fully elongated bristles either the bundles decrease in size in going from the base to the tip or they decrease in number, or both. Certainly, they decrease in number because, in general, 11 bundles are present in cross sections of the microchaete base yet only seven or less near the tip and an intermediate number in sections cut midway along the bristle length. We also know from our thin sections that the bundle size decreases from the base to the tip (Tilney et al., 2000a). How could bundle number be regulated? From our analysis herein (Figure 9), we know that large bundles have proportionately more filaments that are maximally crossbridged to each other than smaller bundles. This is due to the fact that small bundles have a greater surface-to-volume ratio than large bundles (Figure 8). In short, if we combine the facts that attachment of filaments to the plasma membrane and that maximum cross-bridging between filaments in bundles leads to greater stability, then filaments in large bundles should turn over less frequently than those in small bundles. This ultimately should lead to a few large bundles attached to the plasma membrane rather than many small bundles. However, if small bundles were somehow stabilized, then more bundles should occur in our cross sections. It is interesting to note, therefore, that if cytochalasin is added to elongating bristles, the bundle number increases but the size decreases. Our interpretation of this result is that the cytochalasin by capping the barbed ends of the filaments in effect stabilizes these small bundles.

Why have as many as 7 or 11 bundles? Why not just one or two very large bundles? We believe the answer to this question comes from careful examination of the stages in bundle formation. At the extreme bristle tip of an elongating bristle, we find numerous, e.g., two dozen tiny bundles (Tilney et al., 1996) derived from microvilli (DeRosier and Tilney, 2000; our unpublished data). These tiny bundles aggregate into the 7-11 large, approximately equally spaced bundles. This number of bundles seems to be proportional to the distance between the 7-11 resultant cortical bundles. Thus, for aggregation without turnover to occur, the resultant cortical bundles have to be close enough to be aggregated by the forked cross-bridges (Tilney et al., 1998) and accordingly, the bundles will space themselves at intervals dependent upon this distance. If too far apart, they will not aggregate effectively.

\section{How Actin Dynamics Could Influence Bundle Size and Shape}

We've argued that given the membrane surface available for bundle attachment is fixed yet some mutants contain different numbers of actin filaments in each bundle, the shape of the resultant bundles will be dependent on filament number (Tilney et al., 2000b). Accordingly, in a mutant lacking the forked cross-bridge and thus containing fewer filaments than the wild type, the bundle attached to the plasma membrane will be planar or rectangle shaped with the flat side of the rectangle attached to the plasma membrane. Alternatively, if filament number greatly exceeds the number attached to the plasma membrane, then the bundles become triangular or rounded, as in the wild-type or very large and rectangular with the small edge of the rectangle attached to the plasma membrane, as seen in mutants containing additional forked genes or in Stubble or javelin-like mutants (this report). Our interpretation, which is presented for the first time herein, is that if the filaments in the bundles are poorly cross-bridged to each other, they will not be stabilized so that turnover will occur leaving membrane attached filaments and/or ones immediately attached to them. In short, other filaments that are transiently attached to the bundle will be "eaten away" by turnover. Therefore, in the forked mutant the bundle will be thin and flat as indeed they are. In the extreme case where both fascin and the forked proteins are not expressed, e.g., the singed-forked double mutant, all that is seen are membrane attached rafts consisting of a monolayer of actin filaments.

\section{How Actin Dynamics Could Influence Bundle Location}

In the wild type and in most of our mutants, the 7-11 actin cables are spaced at approximately equal intervals. How can one account for this, particularly as there are on average 11 bundles at the bristle base yet only seven at the tip? The tip of a newly emerging bristle has a small diameter yet this tip will ultimately expand into a larger diameter characteristic of the shaft. Why are the bundles at both the base and the tip equally spaced and at the same time occupy a fixed percentage $(7 \%)$ of the shaft cytoplasm (Tilney et al., 2000b)? We believe that the answer may come by studying bristle elongation. We know that the actin cables form by the aggregation of small membrane attached bundles (Tilney et al., 1996). As cross-bridging proceeds, it is sequential first with forked then with fascin. Accordingly, these aggregates gradually increase in size. To achieve equal spacing of bundles, we assume that adjacent bundles "steal" small bundles that lie between each other by aggregation. If small bundles are not aggregated because they are too far from other bundles, being less stable they will turnover. Accordingly, by a mass action process of aggregation and turnover equally spaced bundles will be generated.

\section{How General Is the Concept That Actin Dynamics Help Regulate the Resultant Actin Cytoskeleton?}

There is abundant evidence that actin dynamics plays a regulatory role in the morphogenesis of stereocilia in hair cells of the vertebrate cochlea. For example, the number of stereocilia per hair cell is remarkably constant from cochlea to cochlea, provided the cells being studied in different cochlea are located at the same position in the organ (Tilney et al., 1992a,b). Interestingly, at early stages in the establish- 
ment of the hair cell bundles, the number of stereocilia per cell is 2 to 3 times larger than the final number (Tilney et al., 1992a). Subsequently, stereocilia that are not attached to each other by tip linkages are not incorporated into the staircase array. In essence, unattached stereocilia are labile and resorbed! Because all the stereocilia at an earlier stage contain a bundle of actin filaments, this means certain actin bundles are depolymerized, whereas others increase in length to form the mature stereociliary cluster (Tilney et al., 1992a).

From the literature on the regulation of thin filament length in skeletal muscle (Littlefield et al., 2001), we now recognize that filament length is also influenced by actin dynamics, which is controlled by the relative concentration of pointed end cappers and barbed end cappers.

In all three cases (bristles, stereocilia, and muscle), precise structures are actually the result of a selection, almost a Darwinian selection of more stable patterns of actin filaments over less stable patterns. Thus, the resultant cytoskeleton, which can be reproducible from one cell to the next in these mature differentiated cells, seems to be determined not only by sequential timed steps in morphogenesis but also by turnover leading to a selection of the most stable pattern generated.

This concept is also applicable to controlling the length of microtubule-based cell extensions. Specifically, flagellar length seems to be controlled by tubulin dynamics in which assembly of microtubules in the axonome (and thus increase in flagellar length) is balanced by disassembly, all orchestrated by the movement of tubulin dimers from the base to the tip of the flagellum (Marshall and Rosenbaum, 2001). In an even more general context, the wiring of the nervous system is plastic, in that use specifies the wiring pattern that is generated by controlling turnover of synapses (Katz and Shatz, 1996).

Thus, in biological structures, unlike human-constructed machines like computers, cars, buildings, and bridges, are not built using the same principles. Even though all of these structures are built in sequential steps, biological structures are assembled with plasticity brought about by selecting more stable patterns over less stable patterns. In essence, biology is messy. However, this messiness develops into ordered structures that are unexpectedly complex yet reproduceable and capable of efficiently solving problems that seem at first glance, to be impossible to regulate.

\section{ACKNOWLEDGMENTS}

We thank John Merriam, Vladislav Verkhusha, Nancy Petersen, Ann Hammonds, Jim Fristrom, and the Drosophila Stock Center for generously making fly stocks available. This work was supported by grants from National Institute of Health (GM-52857) to L.G.T and from the National Science Foundation (MCB-0077839) to G.M.G.

\section{REFERENCES}

Appel, L.F., Prout, M., Abu-Shumays, R., Hammonds, A., Garbe, J.C., Fristrom, D., and Fristrom, J. (1993). The Drosophila Stubble-stubbloid gene encodes an apparent transmembrane serine protease required for epithelial morphogenesis. Proc. Natl. Acad. Sci. USA 90, 4937-4941.

Bainbridge, S.P., and Bownes, M. (1981). Staging the metamorphosis of Drosophila melanogaster. J. Embryol. Exp. Morphol. 66, 57-80.

Bartles, J.R., Zheng, L., Li, A., Wierda, A., and Chen, B. (1998). Small espin: a third actin-bundling protein and potential forked protein ortholog in brush border microvilli. J. Cell Biol. 143, 107-119.
Bubb, M.R., Senderowicz, A.M., Sausville, E.A., Duncan, K.L., and Korn, E.D. (1994). Jasplakinolide, a cytotoxic natural product, induces actin polymerization and competitively inhibits the binding of phalloidin to F-actin. J. Biol. Chem. 269, 14869-14871.

Bubb, M.R., Spector, I., Beyer, B.B., and Fosen, K.M. (2000). Effects of jasplakinolide on the kinetics of actin polymerization. An explanation for certain in vivo observations. J. Biol. Chem. 275, 5163-5170.

DeRosier, D.J., and Tilney, L.G. (2000). F-actin bundles are derivatives of microvilli: what does this tell us about how bundles might form? J. Cell Biol. $148,1-6$.

Fei, X., He, B., and Adler, P.N. (2002). The growth of Drosophila bristles and laterals is not restricted to the tip or base. J. Cell Sci. 115, 3797-3806.

FlyBase Consortium. (2002). The FlyBase database of the Drosophila genome projects and community literature. Nucleic Acids Res. 30, 106-108.

Guild, G.M., Connelly, P.S., Vranich, K.A., Shaw, M.K., and Tilney, L.G. (2002). Actin filament turnover removes bundles from Drosophila bristle cells J. Cell Sci. 115, 641-653.

Katz, L.C., and Shatz, C.J. (1996). Synaptic activity and the construction of cortical circuits. Science 274, 1133-1138.

Lindsley, D.L., and Zimm, G.G. 1992. The Genome of Drosophila melanogaster, San Diego: Academic Press.

Littlefield, R., Almenar-Queralt, A., and Fowler, V.M. (2001). Actin dynamics at pointed ends regulates thin filament length in striated muscle. Nat. Cell Biol. 3, 544-551.

Mardahl-Dumesnil, M., and Fowler, V.M. (2001). Thin filaments elongate from their pointed ends during myofibril assembly in Drosophila indirect flight muscle. J. Cell Biol. 155, 1043-1053.

Marshall, W.F., and J. L. Rosenbaum. (2001). Intraflagellar transport balances continuous turnover of outer doublet microtubules: implications for flagellar length control. J. Cell Biol. 155, 405-414

Murphy, D. B. (2001). Fundamentals of Light Microscopy and Electronic Imaging, New York: Wiley-Liss.

Petersen, N.S., Lankenau, D.H., Mitchell, H.K., Young, P., and Corces, V.G. (1994). Forked proteins are components of fiber bundles present in developing bristles of Drosophila melanogaster. Genetics 136, 173-182.

Tilney, L.G., Connelly, P., Smith, S., and Guild, G.M. (1996). F-actin bundles in Drosophila bristles are assembled from modules composed of short filaments. J. Cell Biol. 135, 1291-1308.

Tilney, L.G., Connelly, P.S., Vranich, K.A., Shaw, M.K., and Guild, G.M. (1998). Why are two different cross-linkers necessary for actin bundle formation in vivo and what does each cross-link contribute? J. Cell Biol. 143, 121-133.

Tilney, L.G., Connelly, P.S., Vranich, K.A., Shaw, M.K., and Guild, G.M. (2000a). Actin filaments and microtubules play different roles during bristle elongation in Drosophila. J. Cell Sci. 113, 1255-1265.

Tilney, L.G., Connelly, P.S., Vranich, K.A., Shaw, M.K., and Guild, G.M. (2000b). Regulation of actin filament cross-linking and bundle shape in Drosophila bristles. J. Cell Biol. 148, 87-100.

Tilney, L.G., Cotanche, D.A., and Tilney, M.S. (1992a). Actin filaments, stereocilia and hair cells of the bird cochlea: how the number and arrangement of stereocilia are determined. Development 116, 213-226.

Tilney, L.G., Tilney, M.S., and DeRosier, D.J. (1992b). Actin filaments, stereocilia, and hair cells: how cells count and measure. Annu. Rev. Cell Biol. 8, 257-274.

Tilney, L.G., Tilney, M.S., and Guild, G.M. (1995). F actin bundles in Drosophila bristles. I. Two filament cross-links are involved in bundling. J. Cell Biol. 130, $629-638$.

Verkhusha, V.V., Tsukita, S., and Oda, H. (1999). Actin dynamics in lamellipodia of migrating border cells in the Drosophila ovary revealed by a GFPactin fusion protein. FEBS Lett. 445, 395-401.

Wulfkuhle, J.D., Petersen, N.S., and Otto, J.J. (1998). Changes in the F-actin cytoskeleton during neurosensory bristle development in Drosophila: the role of singed and forked proteins. Cell Motil. Cytoskeleton 40, 119-132.

Zigmond, S.H., Furukawa, R., and Fechheimer, M. (1992). Inhibition of actin filament depolymerization by the Dictyostelium 30,000-D actin-bundling protein. J. Cell Biol. 119, 559-567. 